\title{
Superior Capsule Reconstruction Is a Viable Option for Patients with Symptomatic, Isolated, and Irreparable Supraspinatus Tears
}

\author{
Ben Gabbott, M.R.C.S., Yemi Pearse, F.R.C.S., Magnus Arnander, F.R.C.S., and \\ Duncan Tennent, F.R.C.S.
}

\begin{abstract}
Purpose: To evaluate the outcome of superior capsular reconstruction for isolated subscapularis tears using a decellularized porcine allograft as a superior capsular reconstruction. Methods: Patients who had symptomatic tears of supraspinatus who had failed to improve a conservative rehabilitation program were included. Previous surgery to the shoulder was not a bar to inclusion. Patients were assessed preoperatively and postoperatively with the Oxford Shoulder Score (OSS), the single assessment numeric evaluation (SANE) score, as well as clinically assessed for their shoulder range of motion (ROM). Patients were followed up with in-person clinic appointments for the first year, followed by a postal questionnaire and telephone consultation at a minimum of 2 years following surgery. Results: Twenty patients ( 11 males and 9 females) with a mean age of 67.5 years were included (range: $54-72$ years). The average follow-up period was 31 months (24-50). The mean improvement in OSS was 14 points $(P<.001)$, SANE score was 27 points $(P<.01)$. Significant improvements in mean ROM were recorded (flexion $80.7^{\circ} ; P<.001$; abduction: $81^{\circ} ; P<.001$; external rotation: $31^{\circ} ; P<$ $.001)$. Six patients suffered SCR failures diagnosed by magnetic resonance imaging, despite this they still recorded improvements in recorded OSS, SANE and ROM (excluding external rotation). Patient demographics for failed superior capsule reconstruction (SCR) highlighted smoking and revision surgery as risk factors. Conclusions: SCR appears to be a viable option for patients with symptomatic, isolated, and irreparable tears of the supraspinatus. Our results demonstrate that there are significant improvements in outcome scores and active range of motion, even in the subgroup in whom postoperative structural failure of the SCR was identified. We recommend caution in patients who have previously failed rotator cuff repair and in heavy smokers. Level of Evidence: Level IV, therapeutic case series.
\end{abstract}

\section{Introduction}

$\mathbf{T}$ ears of the superior rotator cuff are common and, if large enough, disrupt the force couples of the glenohumeral joint, resulting in pain and dysfunction. ${ }^{1,2}$ In some cases, the tear is not repairable either because of tendon retraction, tendon quality, or muscle fatty

From the Shoulder Unit, St. George's Hospital, London, United Kingdom. The authors report the following potential conflicts of interest or sources of funding: Y.P., B.G., D.T., and M.A. report fellowship support from Arthrex. D.T. also reports royalties and lecturing fees from Arthrex, outside the submitted work, and holds a patent with ACJ Tightrope. Full ICMJE author disclosure forms are available for this article online, as supplementary material.

Received November 21, 2020; accepted November 22, 2021.

Address correspondence to B. Gabbott, M.R.C.S., Shoulder Unit, St. George's Hospital, Blackshaw Rd London SW17 OEZ.E-mail: Bengabbott@ doctors.org.uk

Crown Copyright (c) 2021 Published by Elsevier Inc. on behalf of the Arthroscopy Association of North America. This is an open access article under the CC BY-NC-ND license (http://creativecommons.org/licenses/by-nc-nd/4.0/). 2666-061X/201843

https://doi.org/10.1016/j.asmr.2021.11.021 atrophy. In these cases, the options are traditionally limited to tendon transfer, in the young and active, ${ }^{1}$ and reverse shoulder arthroplasty. However, with arthroplasty being ill advised under the age of $70,{ }^{3}$ there is a group of patients in whom neither of these treatments offer a great option, with potentially high complication rates. ${ }^{1}$

In 2012, Mihata et al. described the concept of superior capsule reconstruction. ${ }^{4}$ The idea being that by recreating the superior capsule between the glenoid and the greater tuberosity, and then coupling the anterior and posterior rotator cuff to it, the proximal migration of the humeral head can be prevented to allow the restoration of the force couple with the net effect of improving the function of the shoulder. ${ }^{5}$ In 2013, he described the results of this technique using a fascia lata autograft. ${ }^{6-8}$ He subsequently published several papers confirming good results in his expanding patient cohort, with an increased follow-up., 10

Fascia lata grafts are difficult to harvest with the risk of donor site morbidity, ${ }^{11}$ and an alternative was 
sought. Arthrex (Naples, FL) produced DX Matrix, a decellularized porcine allograft, and an arthroscopic technique by which it could be inserted was developed. Given the good results reported by Mihata et al., it was felt that this technique may provide a viable option for this difficult to treat subpopulation. ${ }^{12}$

The purpose of the study was to evaluate the outcome of superior capsular reconstruction for isolated subscapularis tears using a decellularized porcine allograft as a superior capsular reconstruction. Our hypothesis, SCR provides a treatment option for patients with isolated tears of supraspinatus, who were at the upper end of the age range where tendon transfer would be considered but were below the age considered advisable for RSTR, a range of 60-74 years.

The primary aim of the study was a SANE score and OSS that achieved a mean clinically important difference (MCID).

\section{Methods}

Institutional Review Board approval was not required for this study, as the technique is already previously documented and established. This body of work was considered an audit of its implementation into a new trust.

\section{Patient selection}

All patients had symptomatic, degenerative tears of supraspinatus, experiencing symptoms for a minimum of 1 year. On examination of magnetic resonance imaging (MRI), they all demonstrated retraction to the level of the glenoid and Goutalier grade 3 or 4 fatty infiltration of the muscle belly. The subscapularis and infraspinatus were either intact or only partially torn, and therefore, it was believed to be repairable. There was no evidence of glenohumeral arthritis on the MRI scan.

All patients had failed to improve satisfactorily with a therapist-supervised rehabilitation program that included anterior deltoid recruitment, and had sought a surgical option. No other surgery had been undertaken. All patients had received one steroid injection as part of the conservative rehabilitation. As injections may be administered in the community, it was not possible to have full information of any other injections they may have received prior to referral.

All patients were assessed preoperatively with an Oxford Shoulder Score (OSS), the single assessment numeric evaluation (SANE) score, along with a clinical assessment of their shoulder range of motion. The MCID for the OSS is 6 and the SANE is $27 . .^{13,14}$

Patients were followed up with face-to-face clinic appointments for the first year, followed by postal questionnaire and telephone consultation at a minimum of 2 years following surgery. The telephone consultation included a brief history and symptom check. It also included a range of motion examination.

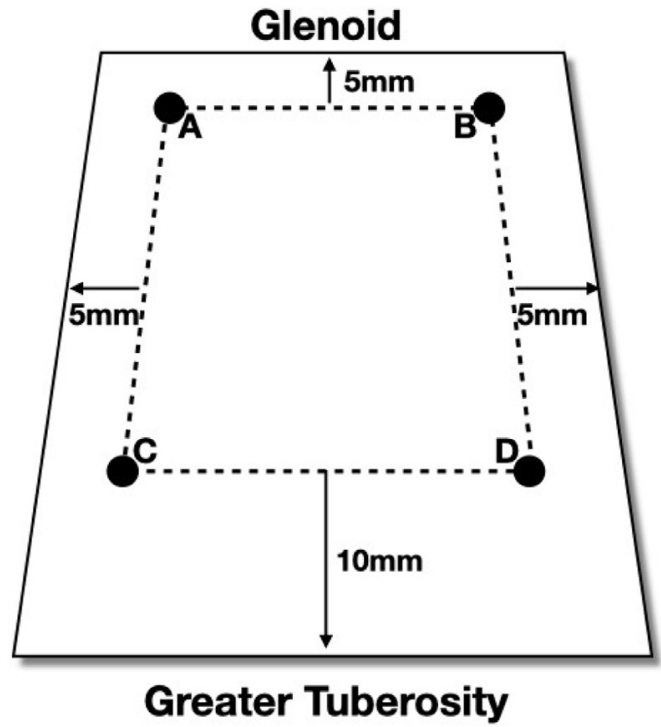

Fig 1. Diagram of the measurements for graft sizing. A-D: graft marks corresponding to anchor positions. A: Posterior glenoid; B: Anterior glenoid; C: Posterior tuberosity medial anchor; D: Anterior tuberosity medial anchor. Measurements indicate the additional size of the graft which was added to the measurements for cutting.

This was achieved by explaining to the patient how a range of motion is measured in a shoulder before asking the patient to perform each movement (abduction / flexion / external rotation / behind the back internal rotation) in front of a mirror and to self-report their active range of motion. If any patient failed to improve, as anticipated, or was considered a potential "failure", MRI scans were obtained, but MRI was not routinely performed on all patients.

All patients were reviewed and recruited preoperatively by the lead surgeon (D.T.). Postoperative data collection was performed by a junior research fellow and a specialist physiotherapist (B.G.).

\section{Surgical Procedure}

All surgery was undertaken by the senior author (D.T.). The patients were placed in the lateral decubitus position with the arm abducted to $30^{\circ}$. All operations were performed under a general anesthetic with an interscalene block. A standard posterior portal was made, and a diagnostic arthroscopy was performed. If a patient was found to have a repairable supraspinatus, then the planned procedure was changed to a repair of the rotator cuff, and these patients were not included in this cohort report. Likewise, If the subscapularis or infraspinatus were found to be irreparable, then no attempt was made to undertake the SCR procedure. Patients who did undergo the SCR technique had the procedure described by Hirahara and Adams ${ }^{15}$ performed.

Using a shaver, the soft tissues over the superior glenoid between 10 and $2 o^{\prime}$ clock and the supraspinatus footprint were debrided, and a biceps tenotomy was 


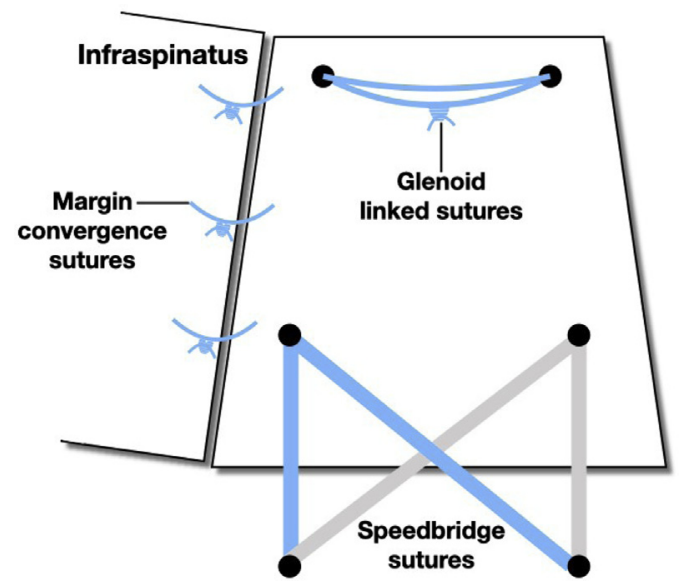

Fig 2. Diagram demonstrating the arrangement of sutures in the final construct, superior view.

performed if the biceps tendon was present. The bone in both regions was then decorticated with the shaver. Two 2.9-mm Biocomposite SutureTak anchors (Arthrex, Naples, FL) were inserted at the anterior and posterior aspects of the prepared glenoid neck $5 \mathrm{~mm}$ from the articular surface. Following this, two 4.75-mm Biocomposite SwiveLock anchors loaded with FiberTape suture (Arthrex) were inserted in the greater tuberosity footprint just behind the articular margin at the anterior and posterior aspects. The distances between the 4 anchors were measured. A margin of $5 \mathrm{~mm}$ was added to the anterior and posterior sides of the glenoid. Ten millimeters was added on the lateral side to complete the sizing (Fig 1). DX Matrix graft was cut to produce 2 pieces of the same size, giving a combined graft thickness of $6 \mathrm{~mm}$. This is comparable to the fascia lata graft, originally described by Mihata et al. ${ }^{7}$ In the first 4 cases, a circumferential 2.0 Vicryl (Ethicon, Edinburgh) mattress stitch was used to produce a double thickness of graft. After this, Dermabond glue (Ethicon, Edinburgh, UK) was used to join the 2 layers. The positions of the suture anchors were marked on the graft.

The glenoid sutures were then retrieved through a laterally placed Passport cannula (Arthrex), and these were passed as an anterior and posterior pair through the graft in the premarked positions. One limb from the anterior anchor was tied to one limb from the posterior anchor and, by pulling on the free limbs, the graft was drawn into the joint. After the first couple of cases, a traction suture was added to the front and back of the graft to aid in the introduction and control the graft in the joint. Once the graft was seated on the glenoid, the free limbs were tied.

The paired sutures from the two SwiveLock anchors were then passed through the graft using a scorpion suture passer (Arthrex). It was discovered early that that the previously marked position on the graft often did not correspond to the most appropriate tension, and

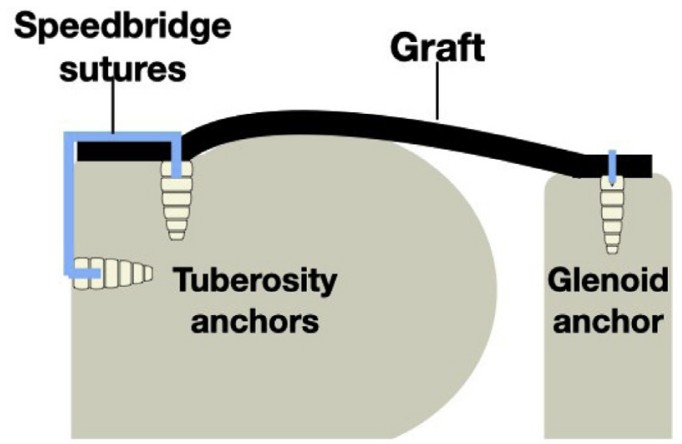

Fig 3. Diagram illustrating the arrangement of anchors and sutures in a lateral view.

therefore, the marks were used as a guide only. The suture pairs were separated. One suture limb from the anterior anchor and one limb from the posterior anchor were then secured laterally using an additional 4.75mm SwiveLock anchor placed on the lateral humeral shaft in line with the previously placed anterior anchor. The process was repeated with an additional posterior anchor in a transosseous equivalent technique (Figs 2 and 3). If there was a tear of either infraspinatus or subscapularis that was repairable, this was performed using the eyelet suture from the medial row anchor. All patients had absent long head of biceps tendon, and no additional procedures were undertaken.

The graft was then secured to the infraspinatus using 3 margin convergence sutures.

Postoperatively the patients were placed in a Donjoy Ultrasling III (DJO, Dallas, TX) for 4 weeks, which provided mild abduction of the arm, after which they started gentle passive range of motion exercises before progressing to active assisted exercises and then full active motion once scapular control had been established. The protocol was the same as that used for typical rotator cuff repairs. Active resistance was not permitted before 3 months.

\section{Results}

Patients were aged 54-74, the patient aged 54 declined tendon transfer and wished to have superior capsule reconstruction. Twenty patients fulfilled the selection criteria and were identified from a specialist shoulder clinic. There were 11 men and 9 women with a mean age of 67.5 years. No patients were excluded.

The patients were reviewed with postal questionnaire and telephone consultation at a mean of 31 months postoperatively (range: $24-50$ ). No patients were lost to follow-up. The data were analyzed using a MannWhitney test (https://www.socscistatistics.com/tests/ mannwhitney/default2.aspx).

The mean preoperative OSS was 24 points (range: 041). The mean improvement in OSS was 14 points (range: $0-43 ; P<.001$. This results in $70 \%$ achieving MCID and $76 \%$ achieving the PASS threshold. The 
Table 1. Outcomes of Superior Capsule Reconstruction at 2 Years

\begin{tabular}{|c|c|c|c|c|c|c|c|}
\hline & Preop Mean (SD) & Preop Range & $\begin{array}{c}\text { Preop Mean } \\
(\mathrm{SD}) \\
\end{array}$ & Preop Range & $\begin{array}{c}\text { Mean } \\
\text { Improvement }(\mathrm{SD})\end{array}$ & Range & $\begin{array}{c}P \text { Value } \\
\text { (Mann-Whitney) }\end{array}$ \\
\hline OSS & $24(12.4)$ & $0-45$ & $38.5(12.5)$ & $0-43$ & $+14(13.4)$ & $0-43$ & $P=.00104$ \\
\hline SANE & $38(22.1)$ & $0-75$ & $70.6(23.8)$ & $25-100$ & $+27(32)$ & $0-82$ & $P=.00614$ \\
\hline Flexion $\left({ }^{\circ}\right)$ & 76.5 & $0-160$ & 159 & $30-180$ & $+81(54)$ & $-15-170$ & $P<.00001$ \\
\hline Abduction $\left({ }^{\circ}\right)$ & 61 & $20-160$ & 154 & $20-180$ & $+82(50)$ & $13-160$ & $P<.00001$ \\
\hline External Rotation $\left({ }^{\circ}\right)$ & 28 & $0-80$ & 54 & $0-90$ & $+31(37.9)$ & $-60-90$ & $P=.01016$ \\
\hline
\end{tabular}

OSS, Oxford Shoulder Score; SANE, Single Assessment Numeric Evaluation; SD, standard deviation.

mean preoperative SANE score was 38 (range: 0-75) and mean postoperative improvement of 27 points (range: $0-82$ ). $P<.01$. This results in $50 \%$ achieving MCID, 38\% reaching SCB, and 50\% achieving the PASS threshold.

The mean active flexion preoperatively was $83^{\circ}$ (range: 30-140) with a mean increased postoperative range of $80.7^{\circ}$ (range: 75-160; $P<.001$. Preoperative abduction was a mean of $76.5^{\circ}$ (range: 0 -160) and postoperatively improved by a mean of $81^{\circ}$ (range: $15-$ 170; $P<.001)$.

Mean preoperative external rotation was $28^{\circ}$ (range: $0-80)$, and postoperatively improved by a mean of $31^{\circ}$ $(-60-90 ; P<.001)$. The full data are presented in Table 1.

\section{Failures}

There were 6 patients classified as failure in this cohort. There are no clear measurements of what constitutes failure; therefore, the reference for this was a comparison with the clinical experience of recovery from a conventional rotator cuff repair. If the patients achieved the expected milestones in their recovery, they were deemed "successful"; if they were failing to achieve the milestones of full active abduction and elevation by 6 months, then the procedure was considered a "failure". In addition, if the patient felt that they had failed to improve to their expectations, the procedure was considered a "failure".

In one patient, the infraspinatus had failed postoperatively, which accounts for the $-60^{\circ}$ improvement in external rotation. She had already undergone a revision of a previously failed rotator cuff repair to an SCR and then lost external rotation, which became apparent 6 months following the procedure. She was subsequently revised to a reverse total shoulder replacement.

One patient had a failure of infraspinatus diagnosed at 4 months following surgery when she lost active external rotation. This was confirmed on an MRI, and she underwent a L'Episcopo latissimus dorsi transfer to restore external rotation. This was performed using the technique described by L'Episcopo et al. for brachial plexus palsy and popularized for rotator cuff failure by Boileau et al. ${ }^{16,17}$ The SCR graft itself was intact.
Two patients failed rapidly, and when the MRI was examined, it was evident that the graft never united with the glenoid in one of the cases, and with the greater tuberosity in the other. Both were heavy smokers (more than 20 cigarettes per day for more than 10 years). Both had also previously failed arthroscopic rotator cuff repair. None of the patients classified as a clinical success smoked.

Two additional patients also "failed". In one patient, the graft did not unite on the glenoid side, and in the other, it failed on the humeral side. Both were revisions from previous unsuccessful rotator cuff repairs, but neither patient smoked (Table 2).

\section{Discussion}

Our study suggests, patients aged 60-74 years, with symptomatic isolated unreconstructable tears of supraspinatus, may have significant improvements in outcome scores and active range of motion following a superior capsule reconstruction.

Mihata et al. first described the biomechanics of the superior capsule in 2012, with subsequent cadaveric studies demonstrating the role of the superior capsule. ${ }^{4,5}$ Further clinical studies by Mihata et al. ${ }^{6,9}$ gave evidence that the theoretical outcomes were supported in clinical practice. Since his original articles were published, there has been an explosion in interest in the procedure. As a consequence of this, 9 modified techniques have been published, ${ }^{18-25}$ which have included different graft materials, the use of the long head of biceps, and partial repair of the retaining cuff over the top of the graft. Since the first clinical article was published in 2013, 5 systematic reviews ${ }^{26-30}$ have also been written. Despite this, it is surprising that there are very few published clinical follow-up studies.

Mihata et al. published an article series with 100 patients followed up to 8 years; however, other authors have not been able to fully replicate his results, and the fascia lata graft itself poses difficulties and complications. ${ }^{9}$ Denard et al. described "satisfactory" results in $70 \%$ of a cohort of 59 patients followed out to a mean of 17.7 months (12-29). ${ }^{31}$ Hiraha et al. reported outcomes in 9 patients at a minimum of 2 years with improvements in ASES and VAS, and there were 2 failures. ${ }^{32}$ Woodmass et al. published the outcomes of 


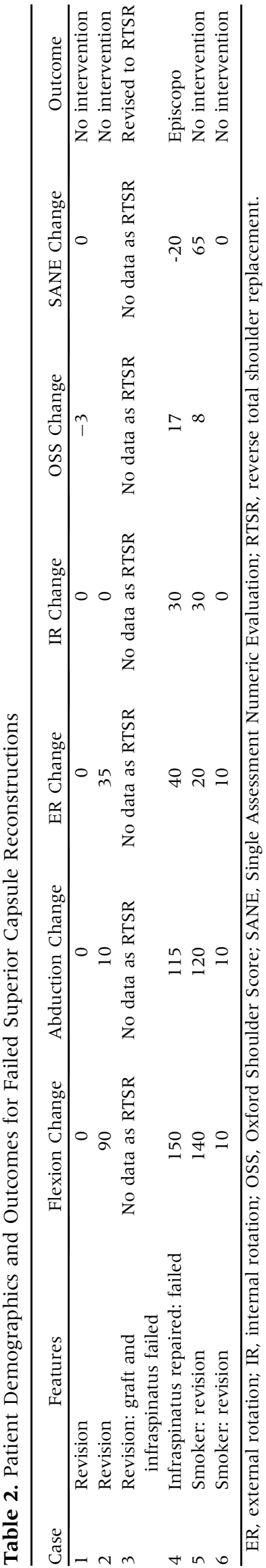

34 patients followed for a minimum of 6 months and a mean of 12 months. They identified 22 failures (66\%). They also identified that subscapularis tears, fatty infiltration of infraspinatus, and revision surgery, as well as female gender were all associated with failure. ${ }^{33}$ Pennington et al. reported outcomes of 88 patients at a minimum 12 month follow-up (16-28 months). They reported $90 \%$ satisfaction with improvement in ASES and VAS scores, as well as range of motion, strength and acromio-humeral distance. In their study, $41 \%$ of patients had undergone previous rotator cuff surgery, and this did not appear to influence the outcome of SCR. Interestingly, the lower limit of the age range was 27 years, with a mean age of 59 , which is considerably lower than the cohort in this series. ${ }^{12}$

This study reports a large cohort with a minimum follow up of 2 years. The results demonstrate that, overall, there are significant improvements in outcome scores and active range of motion, but that the results are not consistent. We identified patients in whom the procedure had failed clinically, and which was confirmed radiologically. In agreement with the Woodmass et al. study, but in contrast to Pennington et al.'s study, 5 of the 6 failures were in patients who had had previous rotator cuff repairs. None of the "clinical" success group had had prior rotator cuff surgery. We found significant improvements even in the group in whom postoperative structural failure of the SCR was identified on MRI; 4 of these 6 patients elected not to undergo any further surgery.

\section{Limitations}

One of the difficulties in analyzing any of these studies is that the reporting is not consistent. Inclusion criteria vary, outcome measures are not uniform, and follow-up is mostly short. There is little radiological review, and the technique has evolved considerably since it was first described. Fascia lata and long head of biceps autograft has both been described, as well as the DX matrix (porcine acellular; Arthrex) and Arthroflex (human acellular; Lifenet, Virginia Beach, FL). In addition, surgeons are using grafts from other sources. Fixation techniques vary as well from the technique described in this article. These include 3 glenoid anchors, knotless versions, and different suture configurations. In addition, there is no agreement on the optimal postoperative regime. All of these confounding factors make comparison of the literature almost impossible.

The use of the xenograft itself may be considered a disadvantage, as it has lower reported healing rates than other sources. At the time of the study, this was the only graft available to us, and we feel that it is worth reporting the outcomes using the graft. We must accept, however, that it should not be inferred that equivalent results will be achieved using allograft. 
There are a number of other limitations to this study. First, there is no control group. As this is a new technique, which was not being proposed as an alternative to any other existing procedure, there was not an obvious control group, and all patients had failed to achieve satisfactory outcomes with nonoperative treatment. This study was designed to assess the feasibility of using this technique in the local population, as all of the published studies at the time were from the originator surgeon. The data also represent a learning curve with the procedure. The senior author had 13 years' experience as an arthroscopic shoulder surgeon and had undertaken the procedure in a cadaveric setting multiple times prior to the study commencing. Even then, refinements were made as experience evolved (for example, the use of traction sutures and the placing of the greater tuberosity anchor sutures). The authors do not believe that this detracts from the findings of the study. No routine postoperative imaging was undertaken, and this is recognized as a limitation, but it was not felt economically justifiable to perform MRIs on all of the patients. Our study also has a small sample size; however, it is in keeping with the sample sizes of other published comparable articles.

\section{Conclusion}

SCR appears to be a viable option for patients with symptomatic, isolated, irreparable tears of the supraspinatus. Our results demonstrate that there are significant improvements in outcome scores and active range of motion, even in the subgroup in whom postoperative structural failure of the SCR was identified. We recommend caution for use in patients who have previously failed rotator cuff repair and in heavy smokers.

\section{References}

1. Greenspoon JA, Petri M, Warth RJ, Millett PJ. Massive rotator cuff tears: Pathomechanics, current treatment options, and clinical outcomes. J Shoulder Elbow Surg 2015;24:1493-1505.

2. Wolf EM, Pennington WT, Agrawal V. Arthroscopic rotator cuff repair: 4-to 10-year results. Arthroscopy 2004;20: 5-12.

3. Guery J, Favard L, Sirveaux F, Oudet D, Mole D, Walch G. Reverse total shoulder arthroplasty. Survivorship analysis of eighty replacements followed for five to ten years. J Bone Joint Surg Am 2006;88:1742-1747.

4. Mihata T, McGarry MH, Pirolo JM, Kinoshita M, Lee TQ. Superior capsule reconstruction to restore superior stability in irreparable rotator cuff tears: A biomechanical cadaveric study. Am J Sports Med 2012;40:2248-2255.

5. Ishihara Y, Mihata T, Tamboli M, et al. Role of the superior shoulder capsule in passive stability of the glenohumeral joint. J Shoulder Elbow Surg 2014;23:642-648.
6. Mihata T, Lee TQ, Watanabe C, et al. Clinical results of arthroscopic superior capsule reconstruction for irreparable rotator cuff tears. Arthroscopy 2013;29:459-470.

7. Mihata T, McGarry MH, Kahn T, Goldberg I, Neo M, Lee TQ. Biomechanical effect of thickness and tension of fascia lata graft on glenohumeral stability for superior capsule reconstruction in irreparable supraspinatus tears. Arthroscopy 2016;32:418-426.

8. Mihata T, McGarry MH, Kahn T, Goldberg I, Neo M, Lee TQ. Biomechanical role of capsular continuity in superior capsule reconstruction for irreparable tears of the supraspinatus tendon. Am J Sports Med 2016;44: 1423-1430.

9. Mihata T, Lee TQ, Itami Y, Hasegawa A, Ohue M, Neo M. Arthroscopic superior capsule reconstruction for irreparable rotator cuff tears: A prospective clinical study in 100 consecutive patients with 1 to 8 years of follow-up. Orthop J Sports Med 2016:4(3_suppl3):2325967116S00076.

10. Mihata T, Lee TQ, Fukunishi K, et al. Return to sports and physical work after arthroscopic superior capsule reconstruction among patients with irreparable rotator cuff tears. Am J Sports Med 2018;46:1077-1083.

11. Walter AJ, Hentz JG, Magrina JF, Cornella JL. Harvesting autologous fascia lata for pelvic reconstructive surgery: Techniques and morbidity. Am J Obstet Gynecol 2001;185: 1354-1359.

12. Pennington WT, Bartz BA, Pauli JM, Walker CE, Schmidt W. Arthroscopic superior capsular reconstruction with acellular dermal allograft for the treatment of massive irreparable rotator cuff tears: Short-term clinical outcomes and the radiographic parameter of superior capsular distance. Arthroscopy 2018;34:1764-1773.

13. Gowd AK, Charles MD, Liu JN, et al. Single assessment numeric evaluation (SANE) is a reliable metric to measure clinically significant improvements following shoulder arthroplasty. J Shoulder Elbow Surg 2019;28:2238-2246.

14. Dawson J, Rogers K, Fitzpatrick R, Carr A. The Oxford shoulder score revisited. Arch Orthop Trauma Surg 2009;129:119-123.

15. Hirahara AM, Adams CR. Arthroscopic superior capsular reconstruction for treatment of massive irreparable rotator cuff tears. Arthrosc Tech 2015;4:e637-e641.

16. L'Episcopo JB. Tendon transplantation in obstetrical paralysis 1934. Am J Surg 1934;25:122-125.

17. Boileau P, Rumian AP, Zumstein MA. 2010. Reversed shoulder arthroplasty with modified L'Episcopo for combined loss of active elevation and external rotation. J Shoulder Elbow Surg 2010;19:20-30.

18. Sutter EG, Godin JA, Garrigues GE. All-arthroscopic superior shoulder capsule reconstruction with partial rotator cuff repair. Orthopedics 2017;40:e735-e738.

19. Kim YS, Lee HJ, Park I, Sung GY, Kim DJ, Kim JH. Arthroscopic in situ superior capsular reconstruction using the long head of the biceps tendon. Arthrosc Tech 2018;7:e97-e103.

20. Petri M, Greenspoon JA, Millett PJ. Arthroscopic superior capsule reconstruction for irreparable rotator cuff tears. Arthrosc Tech 2015;4:e751-e755.

21. Boutsiadis A, Chen S, Jiang C, Lenoir H, Delsol P, Barth J. Long head of the biceps as a suitable available local tissue 
autograft for superior capsular reconstruction: "The Chinese Way". Arthrosc Tech 2017;6:e1559-e1566.

22. Chillemi C, Mantovani M, Gigante A. Superior capsular reconstruction of the shoulder: The ABC (arthroscopic biceps chillemi) technique. Eur J Orthop Surg Traumatol 2018;28:1215-1223.

23. Narvani AA, Imam MA, Polyzois I, et al. The "pull-over" technique for all arthroscopic rotator cuff repair with extracellular matrix augmentation. Arthrosc Tech 2017;6: e679-e687.

24. Cabarcas BC, Garcia GH, Gowd AK, Liu JN, Romeo AA. Arthroscopic superior capsular reconstruction and overthe-top rotator cuff repair incorporation for treatment of massive rotator cuff tears. Arthrosc Tech 2018;7:e829-e837.

25. Burkhart SS, Denard PJ, Adams CR, Brady PC, Hartzler RU. Arthroscopic superior capsular reconstruction for massive irreparable rotator cuff repair. Arthrosc Tech 2016;5:e1407-e1418.

26. Catapano M, Ekhtiari S, Lin A, Bedi A, Lesniak BP. Arthroscopic superior capsular reconstruction for massive, irreparable rotator cuff tears: A systematic review of modern literature. Arthroscopy 2019;35:1243-1253.

27. Ekhtiari S, Adili AF, Memon M, et al. Sources, quality, and reported outcomes of superior capsular reconstruction: A systematic review. Curr Rev Musculoskel Med 2019;12:173-180.

28. Zastrow RK, London DA, Parsons BO, Cagle PJ. Superior capsule reconstruction for irreparable rotator cuff tears: A systematic review. Arthroscopy 2019;35:2525-2534.

29. Dimock RA, Malik S, Consigliere P, Imam MA, Narvani AA. Superior capsule reconstruction: What do we know? Arch Bone Jt Surg 2019;7:3.

30. Wall KC, Toth AP, Garrigues GE. How to use a graft in irreparable rotator cuff tears: A literature review update of interposition and superior capsule reconstruction techniques. Curr Rev Musculoskelet Med 2018;11: 122-130.

31. Denard PJ, Brady PC, Adams CR, Tokish JM, Burkhart SS. Preliminary results of arthroscopic superior capsule reconstruction with dermal allograft. Arthroscopy 2018;34: 93-99.

32. Hirahara AM, Andersen WJ, Panero AAJ. Superior capsular reconstruction: Clinical outcomes after minimum 2-year follow-up. Am J Orthop 2017;46:266-272.

33. Woodmass JM, Wagner ER, Borque KA, Chang MJ, Welp KM, Warner JJ. Superior capsule reconstruction using dermal allograft: Early outcomes and survival. J Shoulder Elbow Surg 2019;28:S100-S109. 